\title{
An Efficient and Accurate Lattice for Pricing Derivatives under a Jump-Diffusion Process
}

\author{
Yuh-Dauh Lyuu \\ Professor and Department Chairman \\ Department of Computer Science \& Information Engineering \\ Department of Finance \\ National Taiwan University \\ Joint work with Tian-Shyr Dai and Chuan-Ju Wang
}




\section{Outline}

(1) Introduction

(2) Models and Definitions

(3) Preliminaries

(4) Lattice Construction

(5) Numerical Results

(6) Conclusion 


\section{Introduction}

- A derivative product is financial instrument whose payoff is based on other underlying assets such as stocks.

- Pricing it is equivalent to computing its expected payoff under a suitable probability measure.

- Most derivatives have no analytical formulas.

- So they must be priced by numerical methods like the lattice model. 


\section{Introduction}

- However, the nonlinearity error may cause the pricing results to converge slowly.

- It may even cause the pricing results to oscillate significantly. ${ }^{1}$

- The goals are numerical accuracy and speed.

${ }^{1}$ Figlewski and Gao (1999). 


\section{Oscillations}

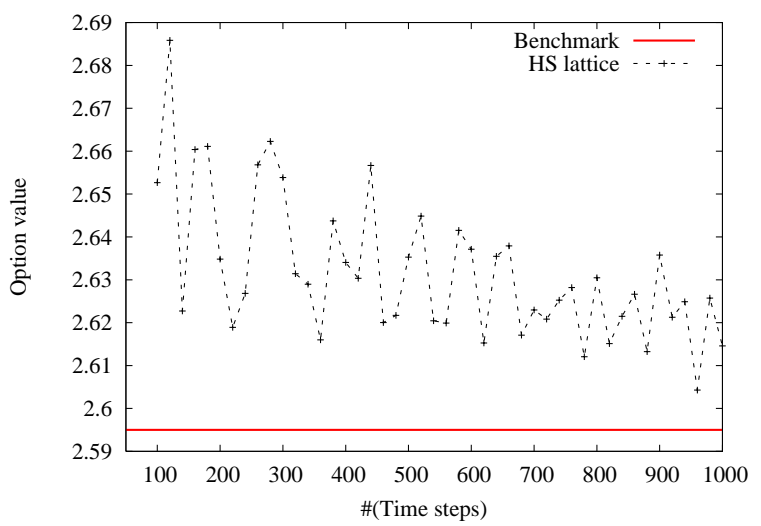




\section{Models}

- Lognormal diffusion process has been widely used to model the underlying asset's price dynamics. ${ }^{2}$

- Unfortunately, the lognormal diffusion process is incapable of capturing empirical stock price behaviors. ${ }^{3}$

- Many alternative processes, such as GARCH process, jump-diffusion process, have been proposed.

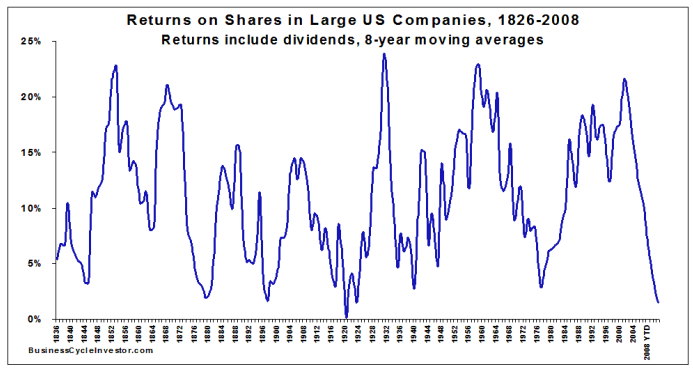

${ }^{2}$ Black and Scholes (1973). 


\section{Related Work}

- Amin (1993)

- He approximates the jump-diffusion process by a multinomial lattice.

- Huge numbers of branches at each node make the lattice inefficient.

- Hilliard and Schwartz (2005)

- They match the first local moments of the lognormal jumps.

- Their lattice lacks the flexibility to suit derivatives' specifications. 


\section{Main Results}

- This talk proposes an efficient lattice model for the jump-diffusion process.

- The time complexity of our lattice is $O\left(n^{2.5}\right)$.

- Our lattice is adjusted to suit the derivatives' specification so that the price oscillation problem can be significantly suppressed. 


\section{Jump-Diffusion Process}

- Define $S_{t}$ as the stock price at time $t$.

- Merton's jump-diffusion model assumes that the stock price process can be expressed as

$$
S_{t}=S_{0} e^{\left.\left(r-\lambda \bar{k}-0.5 \sigma^{2}\right) t+\sigma z(t) U(n(t))\right)} .
$$

- $z(t)$ denotes a standard Brownian motion.

- $r$ denotes the risk-free rate.

- $\sigma$ denotes the volatility of the diffusion component of the stock price process.

- $U(n(t))=\prod_{i=0}^{n(t)}\left(1+k_{i}\right)$ and $k_{0}=0$. 


\section{Jump-Diffusion Process}

- Jump events are governed by the Poisson process $n(t)$ with jump intensity $\lambda$.

- The random jump magnitude $k_{i}(i>0)$ follows the equation:

$$
\ln \left(1+k_{i}\right) \sim N(\gamma, \delta),
$$

where $E\left(k_{i}\right) \equiv \bar{k}=e^{\gamma+0.5 \delta^{2}}-1$. 


\section{Jump-Diffusion Process}

- Hilliard and Schwartz decompose $S_{0}$-log-price of $S_{t}$ into the diffusion component and the jump component by rewriting Eq. (1) as follows:

$$
V_{t} \equiv \ln \left(\frac{S_{t}}{S_{0}}\right)=X_{t}+Y_{t}
$$

- The diffusion component

$$
X_{t} \equiv\left(r-\lambda \bar{k}-0.5 \sigma^{2}\right) t+\sigma z(t)
$$

is a Brownian motion.

- The jump component

$$
Y_{t} \equiv \sum_{i=0}^{n(t)} \ln \left(1+k_{i}\right)
$$

is normal under Poisson compounding. 


\section{Financial Background}

- An option is a financial instrument.

- It represents a right to buy the stock for a price (the exercise price $X)$ at the maturity date $T$.

- Options are essential to speculation and the management of financial risk. ${ }^{4}$

- The payoff of a European-style vanilla option at the maturity date $T$ is $\max \left(S_{T}-X, 0\right)$.

- The payoff of an American-style vanilla option at time $t$ $(0 \leq t \leq T)$ is $S_{t}-X$.

${ }^{4}$ Hull (2002). 


\section{Financial Background}

- A barrier option is similar to a vanilla option.

- But the payoff of a barrier option depends on whether the underlying stock's price path ever touches the barrier(s).

- Such options are very popular in the financial market. 


\section{Barrier Options}

\section{Price}

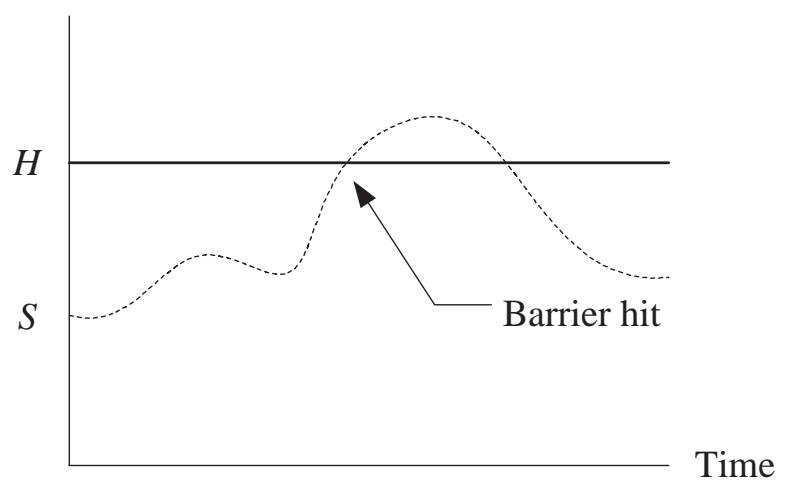




\section{CRR Lattice for the Diffusion Part}

- The size of one time step is $\Delta t=T / n$.

- $u, d, P_{u}, P_{d}$ :

- Match the mean and variance of the stock return.

- $u d=1$.

- $P_{u}+P_{d}=1$.

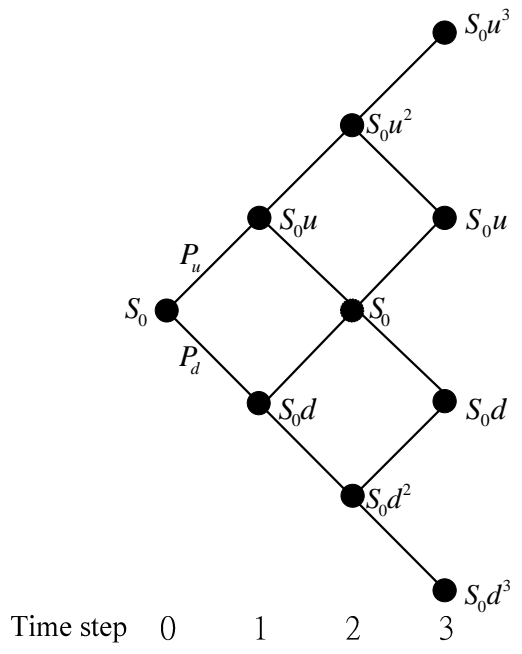




\section{Hilliard and Schwartz's Lattice}

- Diffusion part $\left(X_{t}\right)$

- Match mean and variance of $X_{\Delta t}$.

- Obtain $P_{u}$ and $P_{d}$.

- Jump part $\left(Y_{t}\right)$

- Match the first $2 m$ local moments of $Y_{\Delta t}$.

- Obtain $q_{j}$

$$
(j=0, \pm 1, \pm 2, \ldots, \pm m) \text {. }
$$

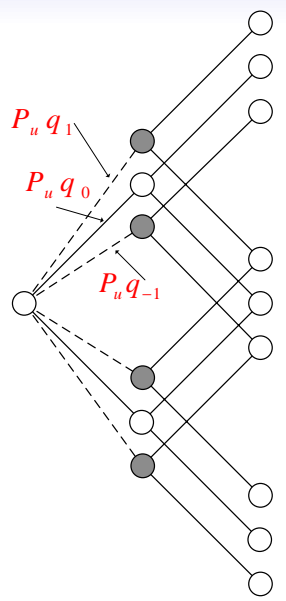




\section{Hilliard and Schwartz's Lattice}

- The stock price of the node at time step $i$ is $S_{0}^{V_{i \Delta t}}$.

- Motivated by the decomposition in Eq. (11), $V_{(i+1) \Delta t}$ can be represented by

$$
V_{(i+1) \Delta t}=V_{i \Delta t}+c \sigma \sqrt{\Delta t}+j h, j=0, \pm 1, \pm 2, \ldots, \pm m
$$

- $c \in\{-1,1\}$ denotes the upward or the downward movement of the stock price driven by the diffusion component.

- $j$ denotes the number of positions above or below the node $V_{i \Delta t}+c \sigma \sqrt{\Delta t}$.

- The magnitude of the basic jump unit is set to $h=\sqrt{\gamma^{2}+\delta^{2}}$.

- The node count of the lattice is $O\left(n^{3}\right)$. 


\section{Hilliard and Schwartz's Lattice}

- Define $F\left(V_{i \Delta t}, i\right)$ as the option value (stock price is $\left.S_{0} e^{V_{i \Delta t}}\right)$.

- European-style options

$$
\begin{aligned}
& F\left(V_{i \Delta t}, i\right)= \\
& e^{-r \Delta t} \sum_{j=-m}^{m} F\left(V_{i \Delta t}+\sigma \sqrt{\Delta t}+j h,(i+1) \Delta t\right) P_{u} q_{j}+ \\
& e^{-r \Delta t} \sum_{j=-m}^{m} F\left(V_{i \Delta t}-\sigma \sqrt{\Delta t}+j h,(i+1) \Delta t\right) P_{d} q_{j} .
\end{aligned}
$$

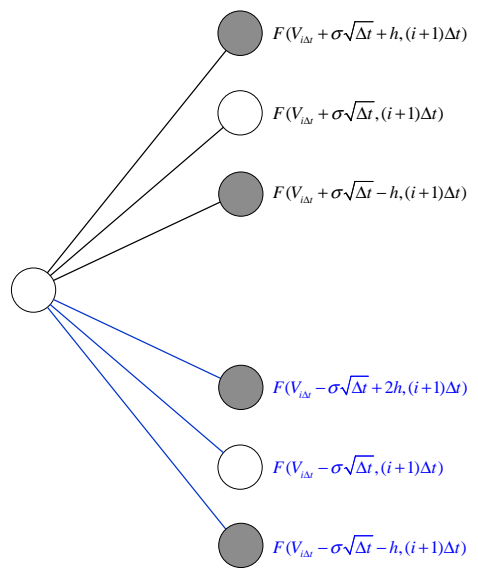




\section{Our Lattice}

- Diffusion part $\left(X_{t}\right)$

- Follow a CRR structure.

- Follow a trinomial structure.

- Jump part $\left(Y_{t}\right)$

- Match the first $2 m$ local moments of $Y_{\Delta t}$.

- Obtain $q_{j}$ $(j=0, \pm 1, \pm 2, \ldots, \pm m)$.

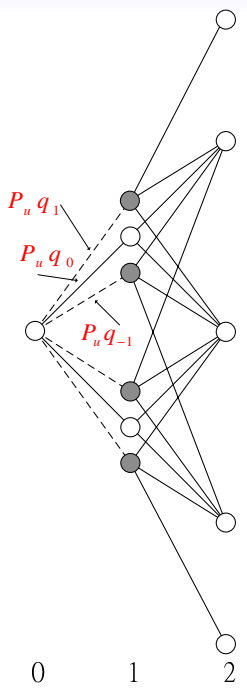




\section{Price Oscillation Problem}

- Price oscillation problem is mainly due to the nonlinearity error.

- Introduced by the nonlinearity of the option value function.

- The solution of the nonlinearity error:

- Making price level of the lattice coincide with the location where the option value function is highly nonlinear.

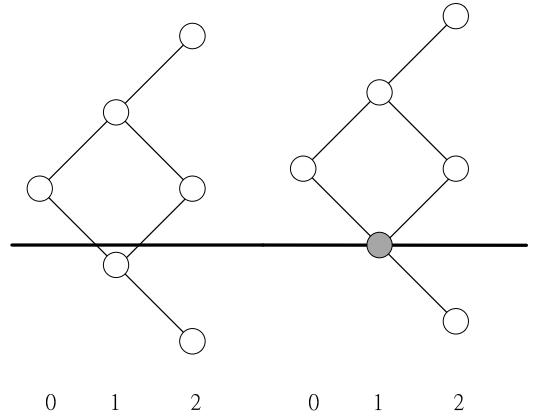




\section{Trinomial Structure}

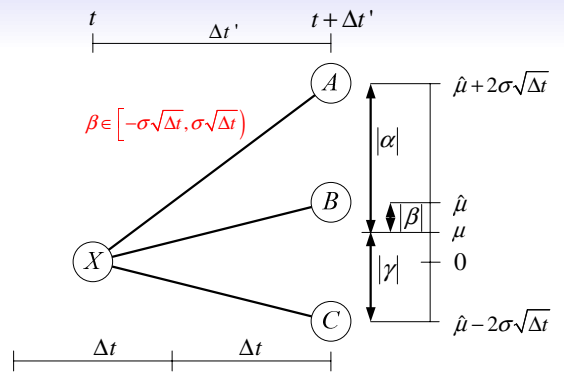

The branching probabilities for the node $X$

$$
\begin{aligned}
& \beta \equiv \hat{\mu}-\mu, \\
& \alpha \equiv \hat{\mu}+2 \sigma \sqrt{\Delta t}-\mu=\beta+2 \sigma \sqrt{\Delta t}, \\
& \gamma \equiv \hat{\mu}-2 \sigma \sqrt{\Delta t}-\mu=\beta-2 \sigma \sqrt{\Delta t}, \\
& \hat{\mu} \equiv \ln (s(B) / s(X)) .
\end{aligned}
$$




\section{Trinomial Structure}

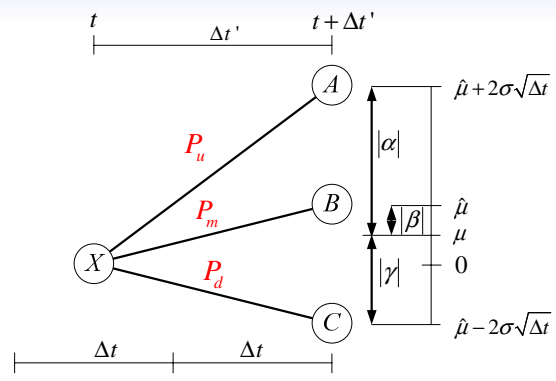

The branching probabilities for the node $X$

$$
\begin{aligned}
P_{u} \alpha+P_{m} \beta+P_{d} \gamma & =0, \\
P_{u}(\alpha)^{2}+P_{m}(\beta)^{2}+P_{d}(\gamma)^{2} & =\text { Var } \\
P_{u}+P_{m}+P_{d} & =1 .
\end{aligned}
$$




\section{Trinomial Structure}

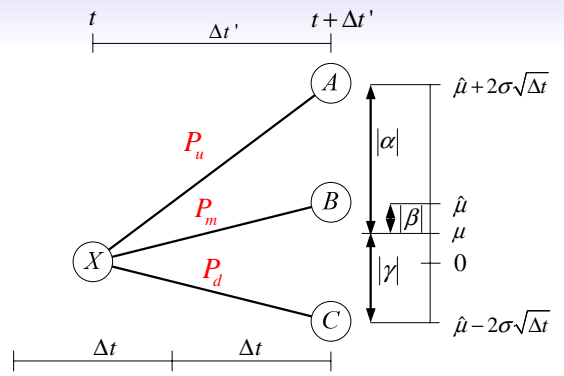

\section{Theorem 1}

Given a node $X$ at time $t$ and a CRR lattice with the length of each time step equal to $\Delta t$ beginning at time $t+\Delta t^{\prime}$, there is a valid trinomial structure from the node $X$ whose $s(X)$-log-price of the central node $B$ lies in the interval $[\mu-\sigma \sqrt{\Delta t}, \mu+\sigma \sqrt{\Delta t})$. Furthermore, the valid branching probabilities can be found by matching the mean and variance of the $s(X)$-log-price of $S_{t+\Delta t^{\prime}}$. 


\section{Adjusting the Diffusion Part of the Lattice}

- Select $\Delta t$ to make $\frac{h^{\prime}-l^{\prime}}{2 \sigma \sqrt{\Delta t}}$ be an integer.

- $\Delta t^{\prime}=T-\left(\left\lfloor\frac{T}{\Delta t}\right\rfloor-1\right) \Delta t$.

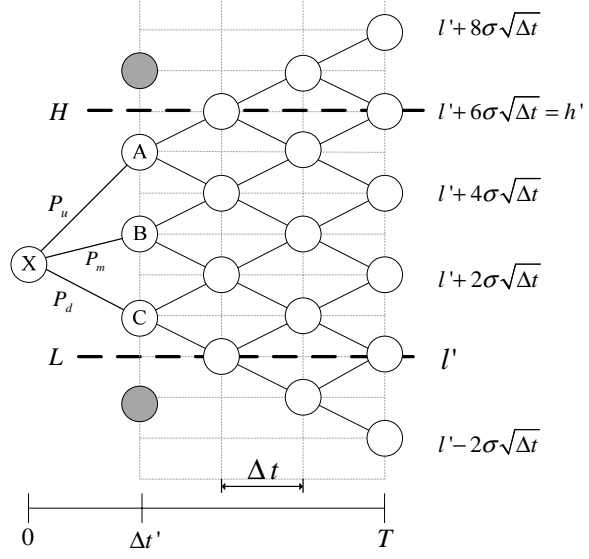




\section{Adjusting the Diffusion Part of the Lattice}

- Lay out the grid from barrier $L$ upward.

- Automatically, barrier $H$ coincides with one level of nodes.

- Obtain $P_{u}, P_{m}, P_{d}$ by Theorem 1 (p. 22).

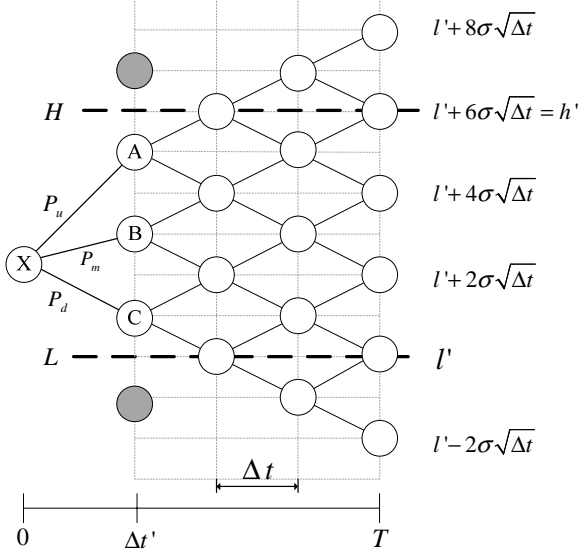




\section{Dealing with Jump Nodes}

- Two phases: the diffusion phase and the jump phase.

- The node count of our lattice is $O\left(n^{2.5}\right)$.

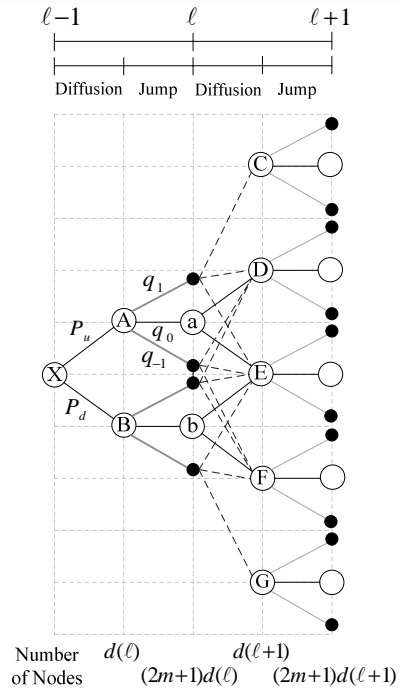




\section{Complexity Analysis}
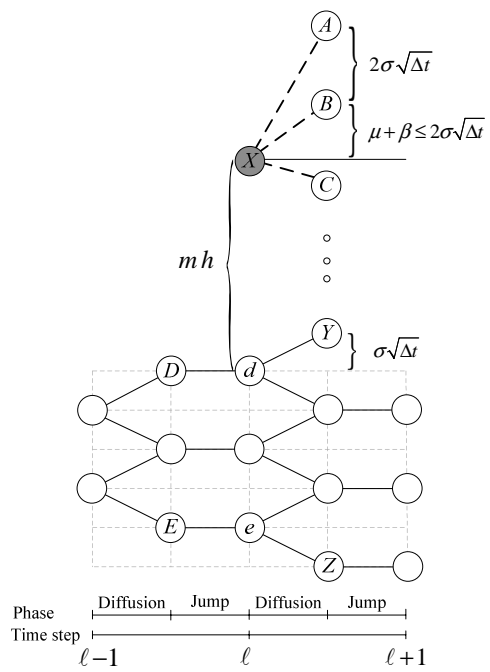

- Node $X$ is the highest jump node at time step $\ell$.

- Node $d$ is the highest diffusion node at time step $\ell$.

- The distance between node $A$ and $Y$ is $<m h+3 \sigma \sqrt{\Delta t}$.

- At each time step, the number of extra diffusion nodes is at most $2\left\lceil\frac{m h+3 \sigma \sqrt{\Delta t}}{2 \sigma \sqrt{\Delta t}}\right\rceil$ (such as nodes $A, B, C$ on the left). 


\section{Complexity Analysis}

- Define $d(\ell)$ as the number of diffusion nodes at time step $\ell$.

- $d(\ell)$ satisfies the following recurrence relation:

$$
\begin{aligned}
d(\ell+1) & =d(\ell)+2\left[\frac{m h}{2 \sigma \sqrt{T / n}}+1.5\right]+1 \\
& =\quad \vdots \\
& =d(1)+2 \times(\ell)\left[\frac{m h}{2 \sigma \sqrt{T / n}}+1.5\right]+\ell, \\
& =O\left(n^{1.5}\right),
\end{aligned}
$$

where $d(1)=2, d(0)=1$, and $\ell \leq n$.

- Consequently, the node count of the whole lattice is $(2 m+1) \sum_{\ell=0}^{n} d(\ell)=O\left(n^{2.5}\right)$. 
Time Complexity

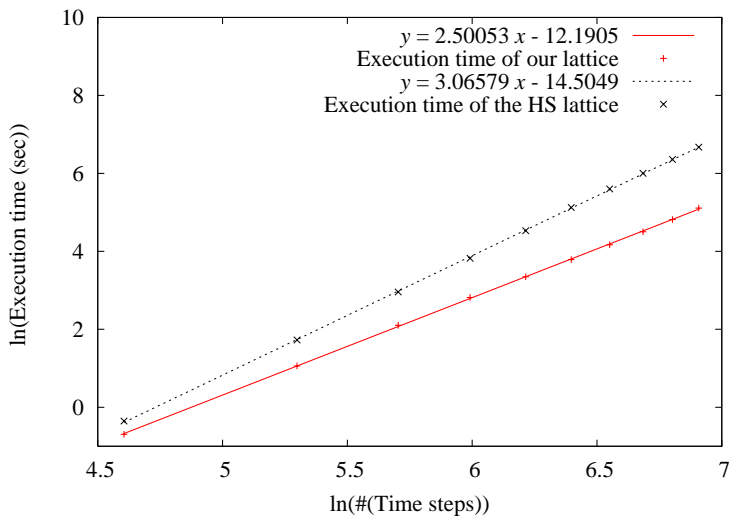

Figure: time complexity. 


\section{Vanilla Options}

\begin{tabular}{|c|c|c|c|c|}
\hline \multirow[b]{2}{*}{ Strike } & \multicolumn{4}{|c|}{ European puts } \\
\hline & Merton & Our Model & $\mathrm{H} \& \mathrm{~S}$ & Amin \\
\hline 30 & 2.172 & 2.194 & 2.189 & 1.837 \\
\hline 35 & 3.810 & 3.793 & 3.788 & 3.553 \\
\hline 40 & 5.980 & 6.002 & 6.004 & 5.783 \\
\hline 45 & 8.650 & 8.630 & 8.638 & 8.501 \\
\hline \multirow[t]{3}{*}{50} & 11.756 & 11.773 & 11.787 & 11.646 \\
\hline & MAE & 0.020 & 0.021 & 0.210 \\
\hline & RMSE & 0.020 & 0.022 & 0.224 \\
\hline & \multicolumn{4}{|c|}{ American puts } \\
\hline Strike & Benchmark & Our Model & $\mathrm{H} \& \mathrm{~S}$ & Amin \\
\hline 30 & 2.279 & 2.278 & 2.272 & 2.057 \\
\hline 35 & 3.972 & 3.971 & 3.965 & 3.878 \\
\hline 40 & 6.308 & 6.313 & 6.313 & 6.249 \\
\hline 45 & 9.163 & 9.164 & 9.165 & 9.157 \\
\hline 50 & 12.560 & 12.562 & 12.566 & 12.559 \\
\hline & $\overline{\mathrm{MAE}}$ & 0.002 & 0.005 & 0.077 \\
\hline & RMSE & 0.002 & 0.006 & 0.111 \\
\hline
\end{tabular}

Table: Pricing European Puts and American Puts. 


\section{Vanilla Options}
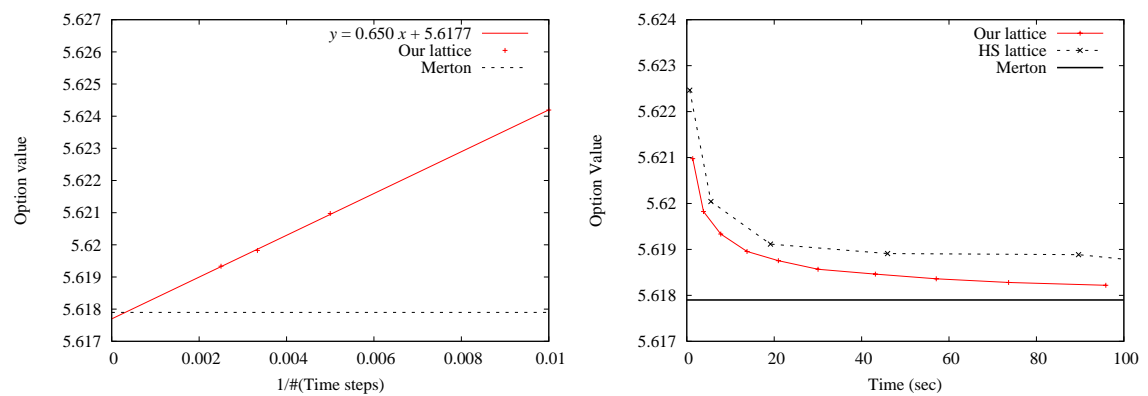

Figure: Converge Property. 


\section{Barrier Options}

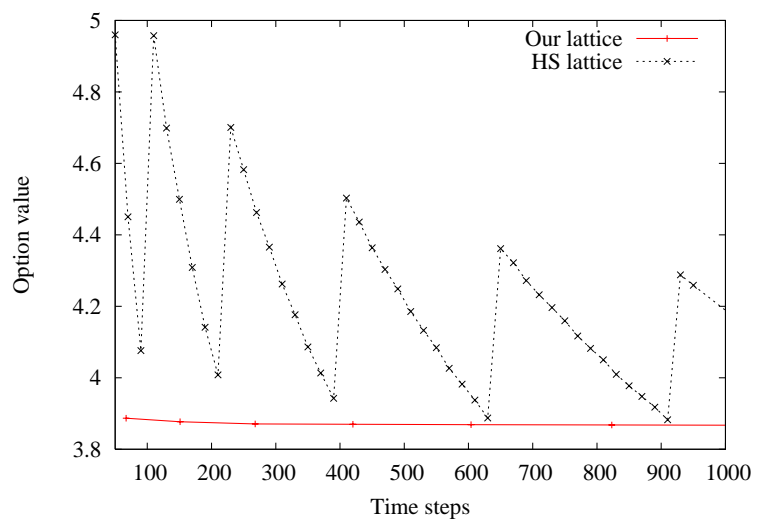

Figure: Pricing a Single-Barrier Call Option. 


\section{Barrier Options}

\begin{tabular}{|c|c|c|c|c|c|}
\hline \multirow[b]{2}{*}{$\begin{array}{l}\text { Time } \\
\text { Steps }\end{array}$} & \multicolumn{3}{|c|}{$\begin{array}{l}\text { European down-and-out and } \\
\text { up-and-out call }\end{array}$} & \multicolumn{2}{|c|}{ Percent errors } \\
\hline & $\begin{array}{c}\text { Simulated Value } \\
(+/-95 \% \text { bounds })\end{array}$ & Our Model & $\mathrm{H} \& \mathrm{~S}$ & Our Model & $\mathrm{H} \& \mathrm{~S}$ \\
\hline 100 & & 2.593 & 2.653 & -0.103 & 2.211 \\
\hline 401 & 2.595 & 2.598 & 2.635 & 0.097 & 1.518 \\
\hline 701 & $(2.584,2.606)$ & 2.599 & 2.621 & 0.125 & 0.989 \\
\hline 1002 & & 2.599 & 2.630 & 0.146 & 1.356 \\
\hline
\end{tabular}

Table: Pricing a Double-Barrier Call Option. 


\section{Conclusion}

- This talk presents a novel, accurate, and efficient lattice model to price a huge variety of derivatives whose underlying asset follows the jump-diffusion process.

- It is the first attempt to reduce the time complexity of the lattice model for the jump-diffusion process to $O\left(n^{2.5}\right)$.

- In contrast, that of previous work is $O\left(n^{3}\right)$.

- With the adjustable structure to fit derivatives' specifications, our lattice model make the pricing results converge smoothly.

- According to the numerical results, our lattice model is superior to the existing methods in terms of accuracy, speed, and generality. 